\title{
A Stopping Rule for Finite-Difference Stochastic Approximation*
}

\author{
Takayuki Wada and Yasumasa Fujisaki \\ Department of Information and Physical Sciences \\ Osaka University \\ 1-5 Yamadaoka, Suita, Osaka 565-0871, Japan \\ E-mail: \{t-wada, fujisaki $\} @$ ist.osaka-u.ac.jp
}

\begin{abstract}
A stopping rule is developed for finite-difference stochastic approximation (FDSA) which is to minimize an unknown objective function based on random noise corrupted observation of the function. When it is assumed that the function is convex quadratic, the necessary number of iterations for achieving a given probabilistic accuracy of the resultant solution is derived, which gives a rigorous stopping rule for FDSA. This number is polynomial in the problem size.
\end{abstract}

\section{Introduction}

Optimization problems with an unknown objective function have many applications in several control synthesis, signal processing, and pattern recognition such as on-line identification, adaptive control, and nonlinear filtering. The Finite-Difference Stochastic Approximation (FDSA) [1,2] is a recursive procedure which has ability to find an optimal solution of an unknown objective function via noisy measurements. Due to the unknown objective function, we have to estimate a gradient of the objective function based on the measurements. The algorithm employs difference quotient to estimate it. Then, the stochastic approximation [3-6] is applied to guarantee validity of this approximation. In fact, convergence to the optimal solution in a probabilistic sense is established in [1,2]. In addition to this, it is important to consider stopping rules for stochastic approximation. For example, in [7-9], it has been shown that the candidate of the solution is close enough to the optimal solution in a practical sense when it satisfies a stopping rule.

In this paper, we develop a stopping rule for FDSA. We give a theoretical guarantee on the stopping rule. We show that the corresponding candidate of the solution is close to the optimal solution within specified estimation error with high probability when the number of iterations approaches to the derived number. Note that once the parameters are specified, the necessary number of the measurements can readily be computed in advance of execution of FDSA. Although the authors have already proposed a stopping rule of linear stochastic approximation for solving an unknown linear equation [10], construction of a stopping rule of FDSA is more difficult than that of the stochastic approximation.

${ }^{*}$ This work was partially supported by JSPS KAKENHI Grant Number 23560530 and 24760344.
This is because the number of parameters of FDSA is greater than that of the stochastic approximation.

This paper is organized as follows. In Section 2, we formulate our problem and show the main result. Then in Section 3, we prove the main result. Finally, we make some concluding remarks in Section 5.

\section{Problem Settings and Main Results}

We consider an unconstrained minimization problem

$$
\min _{x \in \mathbb{R}^{n}} f(x)
$$

where $x \in \mathbb{R}^{n}$ is a decision variable and the objective function $f: \mathbb{R}^{n} \rightarrow \mathbb{R}$ is unknown. FDSA is a kind of gradient methods [11] according to the update rule

$$
x_{k+1}=x_{k}-a_{k} \tilde{g}_{k} .
$$

The gain $a_{k}$ is monotone decreasing sequence which converges to zero as $k$ goes to $\infty$. The vector $\tilde{g}_{k}$ is an estimation of the gradient of $f$ with respect to $x$ at $x_{k}$ based on the difference quotient

$$
\begin{aligned}
\tilde{g}_{k} & =\left[\begin{array}{c}
\tilde{g}_{k, 1} \\
\tilde{g}_{k, 2} \\
\vdots \\
\tilde{g}_{k, n}
\end{array}\right], \\
\tilde{g}_{k, i} & =\frac{y_{k, i}^{+}-y_{k, i}^{-}}{2 c_{k}}, \quad i=1,2, \ldots, n, \\
y_{k, i}^{ \pm} & =f\left(x_{k} \pm c_{k} \psi_{i}\right)+\varepsilon_{k, i}^{ \pm},
\end{aligned}
$$

where $y_{k, i}^{ \pm} \in \mathbb{R}$ are the measurements of $f(x)$ at $x_{k} \pm c_{k} \psi_{i}$, $\psi_{i} \in \mathbb{R}^{n}$ denotes the $i$-th unit vector, and $\varepsilon_{k, i}^{ \pm} \in \mathbb{R}$ are the measurement noise. The step size $c_{k}$ is monotone decreasing sequence which converges to zero as $k$ goes to $\infty$.

In this paper, we assume that the objective function is convex quadratic. This is given by

$$
f(x) \doteq \frac{1}{2} x^{\mathrm{T}} H x+h^{\mathrm{T}} x+d
$$

where coefficients $H=H^{\mathrm{T}}, I / 2 \preceq H \preceq I, h \in \mathbb{R}^{n}$, and $d \in \mathbb{R}$ are unknown. It is supposed that the measurement noise $\varepsilon_{k}^{ \pm}$ are random variables according to the independent and identically distribution with zero mean and the variance $\sigma^{2}<\infty$. 
We select $a_{k}$ and $c_{k}$ as $k^{-1}$ and $k^{-1 / 6}$ respectively, which are the best parameter settings in a theoretical sense [12].

Under these settings, we can construct a stopping rule of FDSA, which is the main result in this paper.

Theorem 1 For given $\alpha \in(0, \infty), \beta \in(0, \infty)$, and $\gamma \in(0,1)$, select $\bar{k} \in \mathbb{N}$ which satisfies

$$
\begin{aligned}
\bar{k} & \geq \max \left\{\tau_{1}, \tau_{2}\right\} \\
\tau_{1} & \doteq\left(\frac{1}{\alpha}\right)^{2}, \\
\tau_{2} & \doteq\left(\frac{3 n \sigma^{2}}{2 \beta^{2} \gamma}\right)^{3 / 2} .
\end{aligned}
$$

Then, for any initial candidate $x_{1} \in \mathbb{R}^{n}$ of the solution, the $\bar{k}$-th candidate $x_{\bar{k}}$ of the solution satisfies

$$
\mathrm{P}^{\infty}\left(\left\|x_{\bar{k}}-x^{*}\right\|_{2} \leq \alpha\left\|x_{1}-x^{*}\right\|_{2}+\beta\right) \geq 1-\gamma,
$$

where $\mathrm{P}^{\infty}$ is a probability measure on a noise sequence and $\|\cdot\|_{2}$ denotes the Euclidean norm.

This theorem says that $x_{\bar{k}}$ is enough close to $x^{*}$ at the $\bar{k}$-th iteration. The distance between $x_{\bar{k}}$ and $x^{*}$ is determined by $\alpha, \beta$, and $\gamma$. These parameters correspond to the initial error, the error caused by noise, and probabilistic guarantee, respectively.

Since $\alpha, \beta$, and $\gamma$ can be chosen as arbitrarily small numbers in this theorem, we have immediately the following corollary which is actually given in a previous work [1].

Corollary 1 For any initial candidate $x_{1} \in \mathbb{R}^{n}$, sequences $x_{1}, x_{2}, \ldots$ of estimations of the solution converges in probability towards $x^{*}$.

\section{Proof of Theorem 1}

We first derive the dynamics of the estimation error $e_{k} \doteq$ $x_{k}-x^{*}$. The gradient estimation $g_{k, i}$ with respect to the $i$-th element of $x$ is

$$
\begin{aligned}
\tilde{g}_{k, i} & =\frac{y_{k, i}^{+}-y_{k, i}^{-}}{2 c_{k}} \\
& =\frac{f\left(x_{k}+c_{k} \psi_{i}\right)-f\left(x_{k}-c_{k} \psi_{i}\right)}{2 c_{k}}+\frac{\varepsilon_{k, i}^{+}-\varepsilon_{k, i}^{-}}{2 c_{k}} \\
& =x_{k}^{T} H \psi_{i}+h^{\mathrm{T}} \psi_{i}+\frac{\varepsilon_{k, i}^{+}-\varepsilon_{k, i}^{-}}{2 c_{k}} .
\end{aligned}
$$

The first-order optimality condition [11]

$$
\frac{\mathrm{d} f}{\mathrm{~d} x}\left(x^{*}\right)=H x^{*}+h=0
$$

leads to $x^{*}=-H^{-1} h$, then we have an expression of the gradient estimation:

$$
\begin{gathered}
\tilde{g}_{k}=\left(x_{k}-x^{*}\right)^{\mathrm{T}} H\left[\begin{array}{llll}
\psi_{1} & \psi_{2} & \ldots & \psi_{n}
\end{array}\right] \\
+\frac{1}{2 c_{k}}\left[\begin{array}{c}
\varepsilon_{k, 1}^{+}-\varepsilon_{k, 1}^{-} \\
\varepsilon_{k, 2}^{+}-\varepsilon_{k, 2}^{-} \\
\vdots \\
\varepsilon_{k, n}^{+}-\varepsilon_{k, n}^{-}
\end{array}\right] \\
=H e_{k}+\frac{\varepsilon_{k}}{2 c_{k}}
\end{gathered}
$$

where we introduce

$$
\varepsilon_{k} \doteq\left[\begin{array}{c}
\varepsilon_{k, 1}^{+}-\varepsilon_{k, 1}^{-} \\
\varepsilon_{k, 2}^{+}-\varepsilon_{k, 2}^{-} \\
\vdots \\
\varepsilon_{k, n}^{+}-\varepsilon_{k, n}^{-}
\end{array}\right]
$$

for readability. The update rule (2) is rewritten as

$$
x_{k+1}=x_{k}-\frac{1}{k}\left(H e_{k}+\frac{\varepsilon_{k}}{2 c_{k}}\right) .
$$

Thus, we immediately see that

$$
e_{k+1}=\left(I-\frac{1}{k} H\right) e_{k}-\frac{1}{2 c_{k} k} \varepsilon_{k}
$$

when we subtract $x^{*}$ from the both sides. Then, the dynamics of the expectation $\mathrm{E}\left[e_{k}\right]$ and the variance $V_{k}$ on $e_{k}$ are

$$
\begin{aligned}
\mathrm{E}\left[e_{k+1}\right]= & \left(I-\frac{1}{k} H\right) \mathrm{E}\left[e_{k}\right] \\
V_{k+1}= & \mathrm{E}\left[\left(e_{k+1}-\mathrm{E}\left[e_{k+1}\right]\right)\left(e_{k+1}-\mathrm{E}\left[e_{k+1}\right]\right)^{\mathrm{T}}\right] \\
= & \left(I-\frac{1}{k} H\right)\left(e_{k}-\mathrm{E}\left[e_{k}\right]\right)\left(e_{k}-\mathrm{E}\left[e_{k}\right]\right)^{\mathrm{T}}\left(I-\frac{1}{k} H\right)^{\mathrm{T}} \\
& +\frac{1}{2 c_{k}^{2} k^{2}} \sigma^{2} I \\
= & \left(I-\frac{1}{k} H\right) V_{k}\left(I-\frac{1}{k} H\right)^{\mathrm{T}}+\frac{1}{2 c_{k}^{2} k^{2}} \sigma^{2} I
\end{aligned}
$$

The solutions of (7) and (8) are given by

$$
\begin{aligned}
\mathrm{E}\left[e_{k}\right] & =\Phi(k, 1) e_{1} \\
V_{k} & =\sum_{j=1}^{k-1} \frac{\sigma^{2}}{2 c_{j}^{2} j^{2}} \Phi(k, j)(\Phi(k, j))^{\mathrm{T}},
\end{aligned}
$$

where $\Phi(k, j)$ is a transition matrix which is defined by

$$
\Phi(k, j) \doteq \begin{cases}\left(I-\frac{1}{k-1} H\right)\left(I-\frac{1}{k-2} H\right) & \\ \quad \times \ldots \times\left(I-\frac{1}{j} H\right) & \text { if } k>j \\ I & \text { if } k \leq j .\end{cases}
$$

Finally, norm of the $k$-th estimation error is bounded by

$$
\begin{aligned}
\left\|e_{k}\right\|_{2} & =\left\|\mathrm{E}\left[e_{k}\right]+e_{k}-\mathrm{E}\left[e_{k}\right]\right\|_{2} \\
& \leq\left\|\mathrm{E}\left[e_{k}\right]\right\|_{2}+\left\|e_{k}-\mathrm{E}\left[e_{k}\right]\right\|_{2} .
\end{aligned}
$$


That is, all we have to do is prove

$$
\begin{aligned}
\left\|\mathrm{E}\left[e_{\bar{k}}\right]\right\|_{2} & \leq \alpha\left\|e_{1}\right\|_{2}, \\
\mathrm{P}^{\infty}\left(\left\|e_{\bar{k}}-\mathrm{E}\left[e_{\bar{k}}\right]\right\|_{2} \leq \beta\right) & \geq 1-\gamma,
\end{aligned}
$$

when the number of iterations reaches $\bar{k}$ which is defined by (3). The following lemmas show that these inequalities actually hold.

Lemma 1 For a given constant $\alpha \in(0, \infty)$, selecting $\bar{k} \in \mathbb{N}$ which is greater than $\tau_{1}$ defined by (4),

$$
\|\Phi(\bar{k}, 1)\|_{2} \leq \alpha
$$

holds, where $\|\cdot\|_{2}$ is the matrix norm induced by the Euclidean norm.

Proof: From the definition of the transition matrix and submultiplicativity of the matrix norm, we obtain

$$
\|\Phi(\bar{k}, 1)\|_{2} \leq \prod_{\ell=1}^{\bar{k}-1}\left\|I-\frac{1}{\ell} H\right\|_{2}
$$

The assumption $I / 2 \preceq H \preceq I$ leads to

$$
\left\|I-\frac{1}{\ell} H\right\|_{2} \leq 1-\frac{1}{2 \ell} \quad \ell=1,2, \ldots, \bar{k}-1 .
$$

Employing

$$
\begin{aligned}
& 1-x \leq \exp (-x), \\
& \sum_{\ell=1}^{k-1} \frac{1}{\ell} \geq \int_{1}^{k} \frac{1}{x} \mathrm{~d} x
\end{aligned}
$$

we see that

$$
\begin{aligned}
\|\Phi(\bar{k}, 1)\|_{2} & \leq \prod_{\ell=1}^{\bar{k}-1}\left(1-\frac{1}{2 \ell}\right) \\
& \leq \prod_{\ell=1}^{\bar{k}-1} \exp \left(-\frac{1}{2 \ell}\right)=\exp \left(-\frac{1}{2} \sum_{\ell=1}^{\bar{k}-1} \frac{1}{\ell}\right) \\
& \leq \exp \left(-\frac{1}{2} \int_{1}^{\bar{k}} \frac{1}{x} \mathrm{~d} x\right)=\sqrt{\frac{1}{\bar{k}}} \\
& \leq \sqrt{\frac{1}{\tau_{1}}}=\alpha .
\end{aligned}
$$

That is, we can conclude that the inequality (9) holds.

Lemma 2 For given constants $\beta \in(0, \infty)$ and $\gamma \in(0,1)$, selecting $\bar{k} \in \mathbb{N}$ which is greater than $\tau_{2}$ defined by (5),

$$
\mathrm{P}^{\infty}\left(\left\|e_{\bar{k}}-\mathrm{E}\left[e_{\bar{k}}\right]\right\|_{2} \leq \beta\right) \geq 1-\gamma
$$

holds.

Proof: Markov inequality [13] leads to

$$
\mathrm{P}^{\infty}\left(\left\|e_{\bar{k}}-\mathrm{E}\left[e_{\bar{k}}\right]\right\|_{2}^{2} \leq \frac{\mathrm{E}\left[\left\|e_{\bar{k}}-\mathrm{E}\left[e_{\bar{k}}\right]\right\|_{2}^{2}\right]}{\gamma}\right) \geq 1-\gamma .
$$

Noting that

$$
\begin{aligned}
& \mathrm{P}^{\infty}\left(\left\|e_{\bar{k}}-\mathrm{E}\left[e_{\bar{k}}\right]\right\|_{2}^{2} \leq \frac{\mathrm{E}\left[\left\|e_{\bar{k}}-\mathrm{E}\left[e_{\bar{k}}\right]\right\|_{2}^{2}\right]}{\gamma}\right) \\
& =\mathrm{P}^{\infty}\left(\left\|e_{\bar{k}}-\mathrm{E}\left[e_{\bar{k}}\right]\right\|_{2} \leq \sqrt{\frac{\mathrm{E}\left[\left\|e_{\bar{k}}-\mathrm{E}\left[e_{\bar{k}}\right]\right\|_{2}^{2}\right]}{\gamma}}\right)
\end{aligned}
$$

and

$$
\begin{aligned}
\mathrm{E}\left[\left\|e_{k}-\mathrm{E}\left[e_{k}\right]\right\| \|_{2}^{2}\right] & =\mathrm{E}\left[\left(e_{k}-\mathrm{E}\left[e_{k}\right]\right)^{\mathrm{T}}\left(e_{k}-\mathrm{E}\left[e_{k}\right]\right)\right] \\
& =\mathrm{E}\left[\operatorname{trace}\left(\left(e_{k}-\mathrm{E}\left[e_{k}\right]\right)\left(e_{k}-\mathrm{E}\left[e_{k}\right]\right)^{\mathrm{T}}\right)\right] \\
& =\operatorname{traceE}\left[\left(\left(e_{k}-\mathrm{E}\left[e_{k}\right]\right)\left(e_{k}-\mathrm{E}\left[e_{k}\right]\right)^{\mathrm{T}}\right)\right] \\
& =\operatorname{trace}_{k},
\end{aligned}
$$

the above probabilistic inequality implies

$$
\mathrm{P}^{\infty}\left(\left\|e_{\bar{k}}-\mathrm{E}\left[e_{\bar{k}}\right]\right\|_{2} \leq \sqrt{\frac{\operatorname{trace} V_{\bar{k}}}{\gamma}}\right) \geq 1-\gamma .
$$

From the solution of $V_{k}, \sqrt{\operatorname{trace} V_{\bar{k}} / \gamma}$ is rewritten by

$$
\begin{aligned}
\sqrt{\frac{\operatorname{trace} V_{\bar{k}}}{\gamma}} & =\sqrt{\frac{1}{\gamma} \operatorname{trace}\left(\sum_{j=1}^{\bar{k}-1} \frac{\sigma^{2}}{2 c_{j}^{2} j^{2}}(\Phi(\bar{k}, j))^{\mathrm{T}} \Phi(\bar{k}, j)\right)} \\
& =\sqrt{\frac{\sigma^{2}}{2 \gamma} \sum_{j=1}^{\bar{k}-1} \frac{1}{c_{j}^{2} j^{2}} \operatorname{trace}\left((\Phi(\bar{k}, j))^{\mathrm{T}} \Phi(\bar{k}, j)\right)} .
\end{aligned}
$$

Similar discussion to the proof of Lemma 1 gives us

$$
\operatorname{trace}\left((\Phi(\bar{k}, j))^{\mathrm{T}} \Phi(\bar{k}, j)\right) \leq \frac{j n}{\bar{k}} .
$$

This estimation leads to

$$
\sqrt{\frac{\operatorname{trace}_{\bar{k}}}{\gamma}} \leq \sqrt{\frac{\sigma^{2}}{2 \gamma} \sum_{j=1}^{\bar{k}-1} \frac{n}{c_{j}^{2} j \bar{k}}}=\sqrt{\frac{n \sigma^{2}}{2 \gamma \bar{k}} \sum_{j=1}^{\bar{k}-1} \frac{1}{j^{2 / 3}}} .
$$

We therefore conclude that the statement of the lemma as

$$
\begin{aligned}
\sqrt{\frac{\operatorname{trace}_{\bar{k}}}{\gamma}} & \leq \sqrt{\frac{n \sigma^{2}}{2 \gamma \bar{k}} \int_{1}^{\bar{k}} x^{-2 / 3} \mathrm{~d} x} \\
& \leq \sqrt{\frac{3 n \sigma^{2}}{2 \gamma \bar{k}^{2 / 3}}} \\
& \leq \sqrt{\frac{3 n \sigma^{2}}{2 \gamma \tau_{2}^{2 / 3}}}=\beta .
\end{aligned}
$$

Theorem 1 is immediately established by these lemmas. That is, from Lemmas 1 and 2 we can see that

$$
\begin{aligned}
\left\|x_{\bar{k}}-x^{*}\right\|_{2}=\left\|e_{\bar{k}}\right\|_{2} & \leq\left\|\mathrm{E}\left[e_{\bar{k}}\right]\right\|_{2}+\left\|e_{\bar{k}}-\mathrm{E}\left[e_{\bar{k}}\right]\right\|_{2} \\
& \leq \sqrt{\frac{1}{\bar{k}}}\left\|e_{1}\right\|_{2}+\sqrt{\frac{3 n \sigma^{2}}{2 \gamma \bar{k}^{2 / 3}}} \\
& \leq \alpha\left\|e_{1}\right\|_{2}+\beta=\alpha\left\|x_{1}-x^{*}\right\|_{2}+\beta
\end{aligned}
$$


with probability $1-\gamma$.

Finally, we hasten to compare difficulty between FDSA and the stochastic approximation (SA). SA is a method for finding a solution of an unknown equation $f(x)=0$ from noisy measurements of its residual. Its update rule is

$$
x_{k+1}=x_{k}-a_{k} y_{k},
$$

where measurement $y_{k}$ of residual is

$$
y_{k}=f\left(x_{k}\right)+\varepsilon_{k},
$$

and $\varepsilon_{k} \in \mathbb{R}^{n}$ is a measurement noise. Now, when we assume that $\varepsilon_{k}$ is a random variable with mean 0 and variance $\sigma^{2} I$, this implies that the mean of $y_{k}$ and its variance are also 0 and $\sigma^{2} I$, respectively. On the other hand, we can interpret solving the optimization (1) as finding $x^{*}$ which satisfies the equation

$$
\frac{\mathrm{d} f}{\mathrm{~d} x}(x)=H x+h=0 .
$$

due to the first order optimality condition. That is, FDSA is a SA for finding the root of the above equation whose measurement corresponds to the gradient estimation

$$
\tilde{g}_{k}=H e_{k}+\frac{\varepsilon_{k}}{2 c_{k}}=\left(H x_{k}+h\right)+\frac{\varepsilon_{k}}{2 c_{k}} \text {. }
$$

Since its variance is $\sigma^{2} /\left(2 c_{k}^{2}\right)$ and $c_{k}$ is monotone decreasing sequence, the variance of the measurement is monotone increasing. We therefore see that construction of a stopping rule of FDSA is more difficult than that of SA.

\section{Numerical Example}

In this section, we investigate the necessary number $\bar{k}$ of iterations of the stochastic approximation. We first prepare some parameters of this algorithm. We give the dimension of $x$ as $n=4$ and we set the variance $\sigma^{2}=0.01$ of $\varepsilon_{k}^{ \pm}$. Setting $\gamma=0.01$, we plot $\tau_{1}$ and $\tau_{2}$ for each $\alpha$ and $\beta$ on Fig. 1 . The solid and the dashed lines denote $\tau_{1}$ and $\tau_{2}$, respectively. From this figure,we see that $\tau_{1}$ and $\tau_{2}$ is a polynomial in $1 / \alpha$ and $1 / \beta$. Furthermore $\tau_{1}$ does not become too large for each $\alpha$. On the other hand, it seems that $\tau_{2}$ becomes large for small $\beta$. Since we do not have to set too small $\beta$ when we utilize FDSA, this issue is not important. For example, if we set $\beta=0.1, \tau_{2} \approx 14,697$ and it is not large.

\section{Concluding Remarks}

In this paper, we have developed a stopping rule for FDSA. Here we have assumed that the objective function is convex quadratic. Then, we have obtained the number of iterations for guaranteeing prespecified quality of the resultant solution. Furthermore, we have clarified that its number is bounded by a polynomial in the problem size. Even if the convex objective function is not quadratic, we may construct a stopping rule based on a similar idea, which is now under investigation.

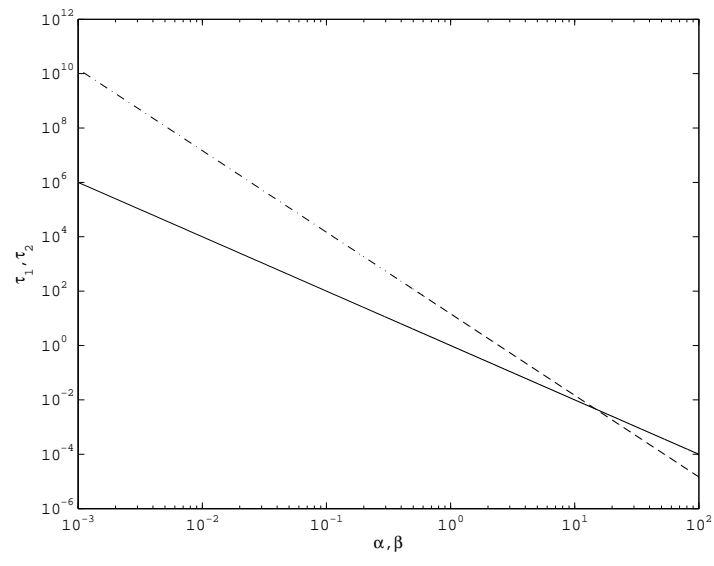

Fig. 1: The necessary number of iterations of FDSA with $\gamma=0.01$ and $\sigma^{2}=0.01$.

\section{References}

[1] J. Kiefer and J. Wolfowitz: Stochastic estimation of the maximum of a regression function; Annals of Mathematical Statistics, Vol. 23, No. 3, pp. 462-466 (1952)

[2] J. R. Blum: Multidimensional stochastic approximation methods; Annals of Mathematical Statistics, Vol. 25, No. 4, pp. 737-744 (1954)

[3] H. Robbins and S. Monro: A stochastic approximation method; The Annals of Statistics, Vol. 22, No. 3, pp. 400-407 (1951)

[4] M. B. Nevelson and R. Z. Hasminskii: Stochastic Approximation and Recursive Estimation; American Mathematical Society (1976)

[5] H. J. Kushner and G. Yin: Stochastic Approximation Algorithms and Applications; Springer-Verlag (1997)

[6] H.-F. Chen: Stochastic Approximation and Its Applications; Kluwer Academic Publishers (2002)

[7] D. W. Hutchison and J. C. Spall: Stochastic approximation in finite samples using surrogate processes; Proceedings of the 43rd IEEE Conference on Decision and Control, pp. 41574162 (2004)

[8] D. W. Hutchison and J. C. Spall: A method for stopping nonconvergent stochastic approximation process; Proceedings of the 44th IEEE Conference on Decision and Control, pp. 6620-6625 (2005)

[9] D. W. Hutchison and J. C. Spall: Stopping small-sample stochastic approximation; Proceedings of 2009 American Control Conference, pp. 26-31 (2009)

[10] T. Wada, T. Itani, and Y. Fujisaki: A stopping rule for linear stochastic approximation; Proceedings of the 49th IEEE Conference on Decision and Control, pp. 4171-4176 (2010)

[11] Y. Nesterov: Introductory Lectures on Convex Optimization: A Basic Course, Vol. 87 of Applied Optimization; Kluwer Academic Publishers (2004)

[12] J. C. Spall: Introduction to Stochastic Search and Optimization; Wiley (2003)

[13] A. N. Shiryaev: Probability; Springer, 2 edition (1996) 